\title{
A Brief Study on Automated Non Contrast Cardiac MRI Segmentation by Machine Vision Techniques
}

\author{
Valliappan Raman, Patrick Then, and Annuar Rapaee
}

\begin{abstract}
Cardiovascular diseases are the major cause of death in the developed world. The high death rate caused by coronary artery diseases increases the need for early detection. Magnetic resonance imaging has turned out to be very promising for this purpose. Currently Gadolinium (contrast agent) is injected intravenously to visualize the accurate myocardial abnormalities. The extent of non-viable tissue in the left ventricle $(\mathrm{LV})$ of the heart is a direct indicator of patient survival rate. The main objective of this paper is to make a review and propose a methodology to segment the non-viable tissue before injecting gadolinium (Contrast agent) in Cardiac MRI by computer vision techniques. The proposed work consists of four stages for an automatic segmentation approach: Data Acquisition, Preprocessing \& motion artifact suppression, Segmentation of the myocardium by healthy and dead area from $L V$ and $R V$ and Classification. This paper investigates and compares the patterns of contrast enhanced and non-contrast enhanced cardiac MRI which causes myocardial abnormalities
\end{abstract}

Index Terms-Myocardium, left and right ventricles, contrast agent, region of interest, segmentation and classification.

\section{INTRODUCTION}

Cardiovascular diseases are the leading cause of death in the world. Many techniques are available to diagnose heart diseases. CT, Nuclear Medicine, Ultrasound and MRI are the most common cardiac imaging techniques [1]. Cardiac magnetic resonance imaging (CMR) is a rapidly evolving field which provides high contrast and high resolution 3-dimensional images of the heart, coronary vessels, and the great vessels without subjecting the patient to ionizing radiation. CMR can provide a comprehensive assessment of the heart including myocardial wall motion, cavity size, ventricular ejection fraction, wall thickness, valvuar function, infarct area, proximal coronary artery lesions, aortic disease including aneurysm and dissection, pericardial disease, congenital heart defects, and myocardial viability. Nonviable myocardium is dead or scar tissue which has permanently lost its function irrespective of its regional blood supply. The LGE signifies the thickness of nonviable or scar tissue and this correlates well to areas that are less viable and are partially or completely damaged in an irreversible manner. Our main objective is to face the challenge of detecting myocardium viability by absence of LGE and to detect the dead tissues from the overall volume of the myocardium.

Manuscript received June 7, 2012; revised July 18, 2012.

Valliappan Raman and Patrick Then are with the Swinburne University of Technology Sarawak (e-mail: vraman@ Swinburne.edu.my, pthen@swinburne.edu.my).

Annuar Rapaee is with the Tropicana Medical Centre (e-mail: annuar.rapaee@gmail.com).

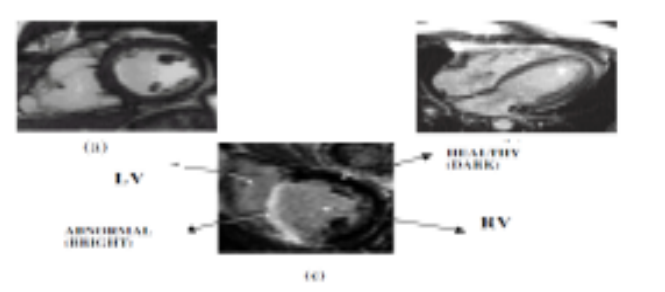

Fig. 1. Illustrates the (a) Cardiac MRI with contrast agent (b) Without contrast agent (C) Myocardium viabilities at left and right ventricles [2]

CMR image analysis has become an active field of research with segmentation and classification of cardiac structures. While performing segmentation, there are many challenges in presence: First endocardium as a boundary between myocardium and blood pool is very hard to be defined, then inducing wrong contours by the heterogeneities in the cavity. Parallel, noticed that the presence of papillary muscles should not be ignored, also the endocardial segmentation should include them into the cavity. Second is the most difficult part of detecting endocardium because of poor edge information in the image between the myocardium and the surrounding anatomical structures such as the liver. The main objective of this paper is to make a review and propose a methodology to segment the non-viable tissue before injecting gadolinium (Contrast agent) in Cardiac MRI by computer vision techniques. Section 1 explains the research challenges in MRI cardiac segmentation; Section 2 explains the focused research objective; existing works are briefly explained in Section 3; Section 4 explains the proposed research methodology for the research problems identified in MRI cardiac segmentation. Finally conclusion is made in Section 5.

\section{RESEARCH CHALLENGES}

MRI datasets are mostly four dimensional $(3 \mathrm{D}+\mathrm{time})$. Manual analysis of these huge datasets is very time consuming. There is few research problems will be focused in this paper. Firstly, the problem is the injection of gadolinium contrast agent in to patient's body and CMR image is taken for detecting the myocardium viability. Cost factor of contrast agent is high, if it undergoes for multiple patients. Also contrast agent leads to varying intensities also. Secondly, in acquisition period the patient is asked to hold the breath. However, not all patients are able to do so during the whole long acquisition period up to 40 seconds and more. This causes motion artifacts based on breathing which have to be detected and compensated. Thirdly, the coronary artery varies from patient to patient. Finally the poor resolution in MRI cardiac images, have noise and poor contrast between myocardium and surrounding tissues makes it difficult to distinguish between them. 


\section{RESEARCH OBJECTIVES}

This paper addresses all of above problems and presents a method to analyze and automatically segment the myocardium by machine vision techniques. First, region of interest (ROI) covering the left cardiac ventricle (LV) is determined. Second image registration is performed to minimize motion artifacts. Finally the myocardium is segmented for analysis. The main objectives are to:

- Reduce the cost with absence of Gadolinium Injection for Cardiac MRI

- Automatic Segmentation of Viable and Non-Viable tissues in Myocardium

- Reducing the noise and increasing the contrast to view myocardium for further analysis

- Detection and Suppuration of motion artifacts

\section{EXISTING WORKS}

There are less number of researches done on detection and segmentation of myocardial viabilities without contrast agents (i.e. absence of gadolinium). But in Cardiac MRI, lot of segmentations and classifications are done by various researchers. Nobel and Hill [3] proposed a method to delineate the borders of the myocardium using non-rigid registration in polar coordinates and to propagate a manual segmentation of a single phase through the dynamic sequence. Lynch [4] used clustering technique based on grey levels to detect the endocardial border. However including the papillary muscles within the endocardial delineation remains a limitation for methods depending on grey level or gradient information. Although techniques using prior knowledge learned from images models to segment the left ventricle. S* orgel et al [5] proposed a method to localize the heart in non-perfusion MRI. This method is used to initialize an active contour approach for segmenting the heart. The images show the heart at different stages of the cardiac cycle. By observing gray value variations over time it is possible to detect the heart. Yang et al [6] have presented a method for compensating translational motion. They conclude to the translational motion using phase differences between successive images.

Spreeuwers and Breeuwer [7] have presented a method detecting myocardial boundaries based on image gray values. This method assumes that no motion artifacts are present or that the images are already registered to each other. They are searching for local maxima in time and space to detect pixels identifying the left and right ventricle. Afterward region growing is applied to extract areas for both ventricles. Then two maximum intensity projections (MIPs) are determined: One for the images before the contrast agent appears in the myocardium, and the other one for the rest of the images. Then these MIP images are subtracted and the myocardium appears as a bright region. Then the image is transformed into polar coordinates and a five node snake is fitted to maximize the contrast ratio. Sun et al [8] have presented a combination of contrast-invariant affine registration and segmentation. This method requires human intervention to select a region of interest (ROI) and a reference image. The registration step is based on edge information's. Therefore edge information's (gradient direction, gradient magnitude) are extracted inside the ROI. A similarity measurement based on these information's is minimized and leads to the final registration. Then for every image the gray value differences to the reference image are calculated. This leads to difference images. Only the ROI is used to gain these difference images. Finally an energy minimization approach is applied to extract the contours of the myocardium.

Santarelli [9] worked on AC models for segmentation. Active contour models have been quite successful for segmenting the myocardium boundaries using CMR images. They still suffer from problems associated with weak gradients between the myocardium and adjacent anatomic structures such as papillary muscles and liver and are sensitive to initialization. Cerqueira et al [10] works divides the myocardium into 17 segments. The heart is divided into three slices (basal, mid-cavity and apical). The basal and mid-cavity slices are divided into six and the apical slice into four segments. The 17 th segment is the apex. Projecting all segments into a single plane leads to the so-called bull's eye plot. Compare to all other works, in this paper, a proposed an automated approach for myocardial viabilities segmentation by computer vision techniques is presented and it will be evaluated by comparison with manual tracing for clinical methods.

\section{RESEARCH METHODOLOGY}

Automated segmentation of the myocardium in cardiac MRI images is quite a difficult challenge. The complexity of this task is based on a wide range of image variabilities. This variability's are caused by patient movement, anatomy variations, pathologies, imaging noise, limited resolution, as well as artifacts. This project presents a method for automatic analysing cardiac MRI data. The designed method is divided into four parts, data acquisition, region identification, segmentation and classification.

\section{A. Data Acquisition}

The CMR image datasets will be acquired at the Sarawak general hospital, in the Department of Cardiology, with the service of radiology section. MRI scans were acquired with an $11.7 \mathrm{~T}, 500 \mathrm{MHz}$ scanner at $26.5 \times 26.5 \times 24.5 \mu \mathrm{m}$ resolution, before histological sectioning.

\section{B. Image Preprocessing}

A pre-processing consists in filtering the original image using alternately area-opening and area closing filters in order to include the papillary muscles into the cavity and to homogenize it. The goal of such filtering is to produce an image that consists mostly of flat and large regions. Connected operators like area opening and closing are useful in such cases. The area opening of an image with defined scale parameters is defined as the image containing connected components of Images with area larger than defined parameters. The area closing is defined by duality. An algorithm developed by D.T Gering [11] can be used to implement these filters on CMR images. 


\section{ROI Determination}

First level is to locate the heart and defines a ROI. After locating the ROI a rigid registration is applied to minimize artifacts caused by patient motion. The main task is to determine the region of interest (ROI). The ROI considered here is the image region covering the left ventricle and the myocardium. A new approach implemented by Zambal et.al [12] is introduced for detecting the heart in MRI. This novel method is based on an incremental pattern matching approach of a very simple pattern of the left ventricle and the myocardium. By observing each time separately motion artifact problems are avoided. This method identifies possible candidates in every single time step using only spatial information. Next step is registration motion pattern searching and reducing motion artifacts, segmentation and classification.

\section{Segmentation}

After registration the segmentation is performed: the goal is to detect the inner and outer boundaries of the myocardium in each time step [13]. The fact that the contrast agent arrives at different anatomical regions at different times allows separating the myocardium from other tissues. The segmentation is done in two steps. In the first step characteristic intensity time curves for four different regions which includes left ventricle, myocardium, right ventricle, and background are calculated. In the second step these curves are used to derive contours for the individual time steps. Finally based on proposed segmentation method, healthy areas indicated by DARK and dead area indicated by BRIGHT will be detected and segmented before applying contrast agent from the LEFT and RIGHT Ventricle area of CARDIAC MRI.

\section{E. Classification}

Once the segmentation is completed, next stage is on Classification. Pixels are classified as belonging either to the left ventricle, myocardium, right ventricle, or background. The characteristic intensity time curves of individual pixels are examined therefore. The characteristic intensity time curve which is most similar defines the class of the pixel. The classification masks obtained in the previous step still contain regions not corresponding to their class. To get a good final estimation of the characteristic intensity time curves these masks have to be refined. The masks of left ventricle and right ventricle are simply refined by selecting their largest connected components

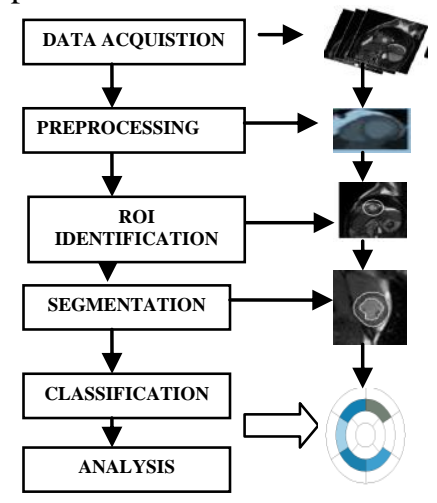

Fig. 2. Illustrates the methodology of myocardium segmentation.

\section{CONCLUSION}

In this paper the automatic segmentation of cardiac MRI data was proposed and discussed. The importance of automatic segmentation of myocardium viabilities without contrast agent by computer vision techniques is discussed. The segmentation of myocardium with darker and brighter areas is highlighted with ROI selection and segmentation methods. Finally it should be remarked that the interpretation of medical images is a highly critical task with respect to a patient's health. Therefore an automatic analysis tool should offer a user interface for the operator to make corrections of automatic generated results possibly at every stage of the process. Currently the work is at proposal stage, in future, it will be implement with further refinements.

\section{ACKNOWLEDGMENT}

We would like to thank Swinburne University of Technology Sarawak for providing facilities to conduct the research and thanks to Dr. Annuar Rapaae, Consultant Cardiologist for providing us more inputs and feedbacks on the proposed framework.

\section{REFERENCES}

[1] R. E. Berbari, I. Bloch, A. Redheuil, E. Angelini, E. Mousseaux, F. Frouin, and A. Herment, "Automated segmentation of the left ventricle including papillary muscles in cardiac magnetic resonance images," FIMH LNCS Proceedings, 2007, vol. 4466, pp 453-462.

[2] P. Viola and W. M.Wells, "Alignment by maximization of mutual information," International Journal of Computer Vision, vol. 24, no. 2, pp.137-154, 1997.

[3] NMI. Noble, DLG. Hill, M. Breeuwer, J. Schnabel, D. Hawkes, FA. Gerritsen, and R. Razavi, "Myocardial delineation via registration in a polar coordinate system," MICCAI LNCS Proceedings, vol. 2488, pp. 651-65, 2002.

[4] M. Lynch, O. Ghita, and PF. Whelam, "Automatic segmentation of the left ventricle cavity and myocardium in MRI data," Journal of Computing Biol Med, vol. 36, pp. 389-407, 2006.

[5] W. S" orgel and V. Vaerman, "Automatic heart localization from a 4D MRI dataset," In Proceedings of SPIE Medical Imaging: Image Processing, vol. 3034, pp. 333-344, 1997.

[6] G. Z. Yang, P. D. Gatehouse, J. Panting, P. Burger, D. J. Pennell, and D N. Firmin, "Motion analysis for magnetic resonance myocardial perfusion imaging," In Proceedings of Image Processing and Its Applications, vol. 2, pp. 838-842, 1997.

[7] L. J. Spreeuwers and M. Breeuwer, "Automatic detection of myocardial boundaries in MR Cardio perfusion images," In Springer Proceedings of Medical Image Computing and Computer-Assisted Intervention, 2001, pp. 1228-123.

[8] Y. Sun, M.-P. Jolly, and J. M. F. Moura, "Contrast-invariant registration of cardiac and renal MR perfusion images," In Springer Proceedings of Medical Image Computing and Computer-Assisted Intervention, 2004, pp. 903-910.

[9] M. F. Santarelli, V. Positano, C. Michelassi, M. Lombardi, L. Landini, "Automated cardiac MR image segmentation: theory and measurement evaluation", Journal of Med Energy Physics, vol. 25, pp. 149-159, 2003.

[10] M. D. Cerqueira, N. J. Weissman, V. Dilsizian, A. K. Jacobs, S. Kaul, W. K.Laskey, D. J. Pennell, J. A. Rumberger, T. Ryan, and M. S. and Verani, "Standardized myocardial segmentation and nomenclature for tomographic imaging of the heart: a statement for healthcare professionals from the cardiac imaging committee of the council on clinical cardiology of the American heart association. Circulation", vol. 5, no. 4, pp.539-542, 2002.

[11] D. T. Gering, "Automatic segmentation of cardiac MRI," In Springer Proceedings of Medical Image Computing and Computer-Assisted Intervention, 2003, pp. 524-532.

[12] S. Zambal, A. Schollhuber, K. B"uhler, and J. Hladuvka, "Fast and robust localization of the heart in cardiac mri series," International Conference on Computer Vision Theory. 
[13] S. C. Mitchell, J. G. Bosch, B. P. F. Lelieveldt, R. J. van der Geest, J. H. C. Reiber, and M. Sonka, "3-D active appearance models: segmentation of cardiac MR and ultrasound images," IEEE Trans. Med. Imaging, vol.21, no. 9, pp.1167-1178, 2002.

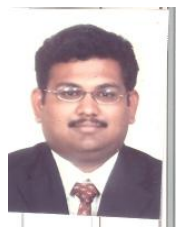

Valliappan Raman is currently doing his $\mathrm{PhD}$ in mammogram tumor segmentation and classification at University Sains Malaysia. He has completed his Master's Degree in 2005 at University Sains Malaysia and completed his Bachelor of Engineering in Computer Science in 2002 at Madurai Kamaraj University. He has been working as lecturer in Swinburne University of Technology Sarawak campus. He have undergone various research projects under major government grants and published papers, articles and journals. His research interest is in medical imaging, watermarking and health informatics

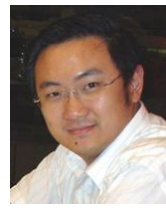

Patrick Then is currently Senior lecturer in Swinburne University of Technology Sarawak Campus. He is the head of Advanced of Informatics Group Lab. He have undergone and headed various research projects funded by Malaysian national grants and published papers in highly repotted journals and conferences. His research area of interest is on watermarking, computer vision and pattern recognition, data mining \& bio-informatics.

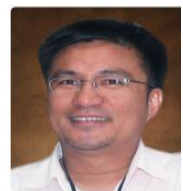

Dr. Annuar Rapaae is currently a consultant cardiologist in Tropicana medical center, Kualalumpur Malaysia. His research interest are more specialized on cardiovascular disease diagnosis, computer aided diagnosis on a cardiac images. 